\title{
Aplicación de herramientas de realidad aumentada a modelos de puentes elaborados con BIM
}

\section{Application of Augmented Reality Tools to Bridge Models Elaborated with BIM}

\author{
Fernando Calderón de Diego ${ }^{*, a}$, Riyadh Hindi ${ }^{\mathrm{b}}$, José Antonio Lozano Galant ${ }^{\mathrm{c}}$ \\ a Ingeniero de Caminos, Canales y Puertos. Universidad de Castilla-La Mancha \\ ${ }^{b}$ Full Professor. Saint Louis University \\ ${ }^{c}$ Dr. Ingeniero de Caminos. Universidad de Castilla-La Mancha
}

Recibido el 30 de mayo de 2019; aceptado el 14 de diciembre de 2020

\section{RESUMEN}

La tecnología evoluciona rápida y constantemente, jugando un papel muy importante en nuestra sociedad, ya que optimiza recursos, tiempo y dinero. En ingeniería civil, el ejemplo más actual es el uso de Building Information Modeling (BIM), una metodología colaborativa para diseñar, construir y administrar más eficientemente. El modelo creado con BIM puede vincularse con Realidad Aumentada (RA), que permite la interacción del usuario con él. La RA superpone ese modelo 3D a la realidad para aumentar la información. Este artículo ilustra el estado actual de la aplicación de BIM y AR en la ingeniería civil, y propone soluciones basadas en un análisis particular: un modelo de puente desarrollado con Revit.

(C)2022 Hormigón y Acero, la revista de la Asociación Española de Ingeniería Estructural (ACHE). Publicado por Cinter Divulgación Técnica S.L. Este es un artículo de acceso abierto distribuido bajo los términos de la licencia de uso Creative Commons (CC BY-NC-ND 4.0)

PALABRAS CLAVE: BIM, realidad aumentada, interoperabilidad, modelo de puente, tecnología, visualización.

\section{ABSTRACT}

Technology evolves rapidly and constantly, and it plays a very important role in our society as it optimizes resources, time and money. In the civil engineering field, the most current example is the use of Building Information Modeling (BIM), a collaborative methodology to design, build and manage more efficiently. The model created with BIM can be linked with Augmented Reality (AR) technology, which allows interaction with it. AR superimposes that 3D model to the real environment to increase the information. This paper illustrates the current state of the applications of BIM and AR into the civil engineering field, and proposes some solutions based on a particular case analysis: a bridge model developed with Revit.

@2022 Hormigón y Acero, the journal of the Spanish Association of Structural Engineering (ACHE). Published by Cinter Divulgación Técnica S.L. This is an open-access article distributed under the terms of the Creative Commons (CC BY-NC-ND 4.0) License

KEYWORDS: BIM, augmented reality, interoperability, bridge model, technology, visualization.

1.

\section{INTRODUCCIÓN}

Hoy día, las tecnologías se encuentran en una constante y rápida evolución, alcanzando todos los campos de estudio e incluso situaciones de nuestra vida cotidiana. Su importancia aumenta notablemente, pues la mayoría sirven para optimizar recursos, tiempo y dinero, por lo que juegan un papel muy importante en la sociedad. En el campo de la ingeniería civil no podría ser

* Persona de contacto / Corresponding author:

Correo-e / e-mail: fernando.calderon@alu.uclm.es (Fernando Calderón de Diego) menos, y por eso surgen nuevas metodologías de trabajo, como Building Information Modeling (BIM).

BIM es una metodología de trabajo colaborativa en la que se crean y gestionan proyectos a través de un modelo 3D digital multidisciplinar. Este modelo conforma una gran base de datos con información, como la geometría del objeto, datos físicos, coste, tiempo de ejecución, datos sobre mantenimiento, etc. Con esta base de datos se puede gestionar cualquier elemento de la infraes- 
tructura durante todo el ciclo de vida de la misma. Además, BIM brinda a todos los profesionales involucrados en el proyecto las herramientas para planificar, diseñar, construir y administrar edificios e infraestructuras de manera más eficiente, lo que se traduce en una reducción tanto en tiempos como en costes.

Además, existen otras tecnologías que mejoran la visualización de elementos y su comprensión, e incluso permiten interactuar con ellos, como la Realidad Aumentada (RA). A pesar de ser relativamente moderna, está bastante implementada en sectores como el turismo o los videojuegos. También se está comenzando a utilizar en estudios de arquitectura y agencias inmobiliarias, ya que es una forma más simple y llamativa de mostrar un producto a un cliente. Sin embargo, en el campo de la ingeniería civil su uso es muy reducido, y se está probando que aumentar su implementación es muy beneficioso, ya que es una forma rápida y eficiente de obtener in situ la información necesaria, de forma visual y precisa, durante toda la vida de la estructura, sin tener que recurrir al proyecto. Algunos de estos ejemplos de interacción BIM-RA son: la creación de aplicaciones con instrucciones para mejorar el mantenimiento de tuberías en instalaciones complejas e informar de protocolos de cierre de tuberías, por Pei-Huang Diao et al.; el desarrollo de una aplicación para facilitar el soporte en las operaciones en obra, por Khaled El Ammari et al.; o la aplicación de RA para crear un sistema de control de proyectos basado en interacciones hombre-máquina para mejorar la toma de decisiones, el proceso de aprobación y la gestión de la información, por Peter E.D. Love et al.

Tanto BIM como RA están aún en fase de desarrollo y la combinación entre ambos ha sido brevemente estudiada, pero está captando la atención del sector de la ingeniería civil y se está generando un creciente interés en los últimos años. El objetivo principal de este artículo es estudiar la viabilidad del uso de la realidad aumentada en modelos de puentes elaborados con Revit, analizando la interoperabilidad entre este y distintos softwares de RA. Tras el proceso de análisis se indicarán los resultados obtenidos, se expondrán una serie de conclusiones y se propondrán posibles trabajos o investigaciones que serían aconsejables llevar a cabo posteriormente.

\subsection{Literatura disponible}

Antes de comenzar a trabajar en la interacción de realidad aumentada y puentes elaborados con BIM, se analizó la literatura disponible y el estado del arte tanto de BIM como de RA. De los análisis podemos extraer como conclusiones que: (1) BIM lleva poco tiempo implementado en la ingeniería civil, pero ya cuenta con un gran papel en ella; (2) con BIM se aceleran los procesos de diseño y construcción, principalmente gracias al trabajo multidisciplinario y la detección de colisiones, lo que se traduce en ahorros económicos y de tiempo; (3) gracias a BIM se puede tener toda la información de cualquier elemento durante todo el ciclo de vida del mismo, lo que facilita su construcción, explotación y reparación o demolición; (4) al trabajar con BIM se crea un modelo 3D que puede ser aprovechado por la RA; (5) el uso de RA facilita la visualización y compresión de cualquier modelo, lo que la hace una buena herramienta en la ingeniería civil; (6) la utilización de RA podría facilitar, principalmente, los procesos de diseño, construcción, explotación y mantenimiento; (7) gracias a la accesibilidad de los dispositivos móviles, implementar RA no supone un gran aumento del coste.

\subsection{Metodología empleada}

Este artículo se ha dividido en 5 secciones, donde la primera es la introducción. Dichas secciones hacen referencia al orden seguido durante el desarrollo del proyecto: en la sección 2 se presentan los programas estudiados, así como las conclusiones obtenidas tras el análisis y la elección del software que se utilizará para elaborar el modelo del puente; en la sección 3 se utiliza el software elegido en el punto anterior para elaborar una aplicación de realidad aumentada para un puente real; en la sección 4 se plantea las posibles líneas de investigación que se podrían seguir tras el presente proyecto; en la sección 5 se exponen las conclusiones finales obtenidas.

Por favor, nótese que en el presente artículo se han descrito muy brevemente algunos de los procesos seguidos para la investigación, y se tiene como objetivo principal reflejar los resultados y conclusiones obtenidos. Para más información puede consultar un vídeo demostrativo del resultado haciendo clic en el siguiente enlace: https://www.youtube.com/watch?time_continue $=3 \& v=j \mathrm{jcdm} 7 \mathrm{WqHA} 4$

\section{2.}

\section{ANÁLISIS DE DISTINTOS PROGRAMAS DE REALIDAD AUMENTADA}

Actualmente, existe una gran cantidad de programas comerciales para trabajar con realidad aumentada. El primer paso fue estudiar cuál de estos programas eran los más utilizados o recomendados. En esta sección se expone el análisis de dichos programas, estudiando en primer lugar las características generales de cada uno y realizando finalmente una comparación de todos ellos siguiendo una estructura académica. Para ello, se ha diseñado un modelo 3D de una estructura sencilla que contiene todos los elementos disponibles en Revit, con el fin de estudiar su interoperabilidad y rendimiento en distintos softwares.

\subsection{Modelo de prueba}

El modelo diseñado para realizar el análisis de los distintos programas está formado por dos pórticos, uno de hormigón y uno metálico, un muro y la topografía. Todo el modelo cuenta con un elevado nivel de detalle, incorporando armaduras y uniones según fuera necesario. El resultado obtenido para el modelo puede apreciarse en la siguiente imagen.

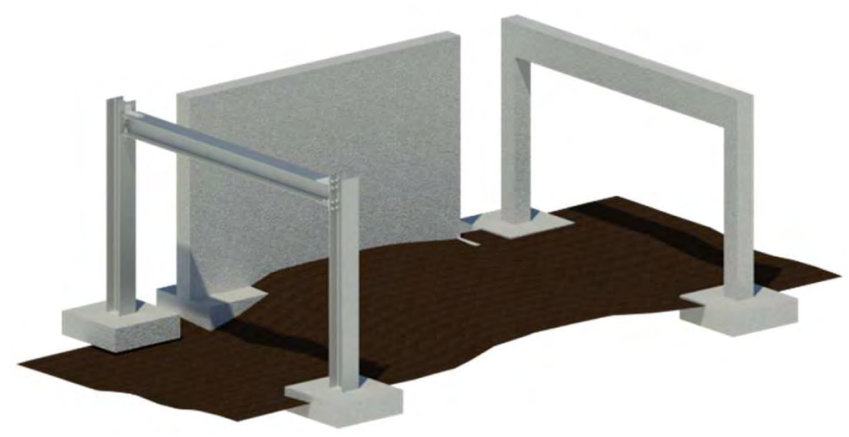

Figura 1. Vista 3D del modelo de prueba. 


\subsection{Programas analizados}

Se han analizado 8 programas distintos, para los cuales se ha estudiado las plataformas en las que operan, las características y limitaciones que plantea cada uno, y el flujo de trabajo que se debe seguir para elaborar el modelo de realidad aumentada. Dichos programas son, ordenados alfabéticamente: (1) ARki; (2) AUGmentecture; (3) Aumentaty (4) UniteAR; (5) Unity; (6) Unreal Engine; (7) WakingApp; y (8) Zappar.

Tras el análisis, se han obtenido unas conclusiones, las cuales se han plasmado en tablas para facilitar la comprensión y comparación entre softwares. La tabla 1 muestra la interoperabilidad por elementos, es decir, lo que cada software de realidad aumentada permite importar. Como el modelo de prueba que se empleó para analizar los softwares cuenta con todos los elementos disponibles en Revit, estos han sido analizados individualmente y representados en columnas. Además, se han habilitado columnas para comprobar si tanto los materiales (hormigón y acero) como la información BIM podría ser importada por cada software. Para poder evaluar esta interoperabilidad se han empleado tres marcadores gráficos: un tick verde, que indica que la interoperabilidad ha sido satisfactoria; un guion amarillo para aquellos elementos de los que no se ha podido obtener información de interoperabilidad; y una cruz roja, para aquellos elementos que no han sido importados con éxito. Los softwares han sido indicados de manera enumerada según un orden alfabético por cuestiones de espacio.
En la tabla 1 se aprecia que no hay información para algunas armaduras. Esto se debe a que en los cuatro primeros softwares no es posible mostrar $\mathbf{u}$ ocultar elementos a voluntad del usuario, con lo que no existe forma de poder ver el interior y poder ver las armaduras. Además, ninguno de los programas muestra información sobre las soldaduras. Esto se debe a que estas no son visibles tampoco en Revit, y por lo tanto no pueden ser importadas a pesar de que el modelo nativo cuenta con dicha información. También se puede observar que Unity y Unreal son los únicos programas que permiten la importación de la información BIM.

La tabla 2 muestra los formatos de archivo que cada programa permite importar y si se necesita de algún software o plug-in intermedio para ello. Unity y Unreal permiten más archivos que los expuestos en la tabla, como 3DS, DXF, MAX o BLEND, pero en ella se han indicado únicamente los principales. De nuevo, para esta tabla se ha utilizado la misma numeración de los programas y el mismo sistema de calificación que para la tabla anterior, a excepción de la columna de "software intermedio", donde se ha utilizado la palabra "no" si no se necesita de un software o plug-in intermedio, y la palabra "si" si se precisa de alguno de ellos.

En la tabla 2 se aprecia cómo Unity y Unreal son los softwares que permiten importar mayor número de formatos de archivo distintos. También se aprecia cómo el formato de archivo FBX (Filmbox), creado por Kaydara y perteneciendo a Autodesk para mejorar la interoperabilidad entre softwares de

TABLA 1.

Interoperabilidad por elementos

\begin{tabular}{|c|c|c|c|c|c|c|c|c|c|c|c|c|}
\hline Software & Terreno & Vigas & Columnas & Paredes & Cimentación & Armadura & Tornillos & Soldaduras & Placas & Hormigón & Acero & Información BIM \\
\hline 1 & $\checkmark$ & $\checkmark$ & $\checkmark$ & $\checkmark$ & $\checkmark$ & - & 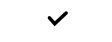 & - & $\checkmark$ & $x$ & $x$ & $x$ \\
\hline 2 & $\checkmark$ & $\checkmark$ & $\checkmark$ & $\checkmark$ & $\checkmark$ & - & $\checkmark$ & - & $\checkmark$ & $\checkmark$ & $\checkmark$ & $x$ \\
\hline 3 & $\checkmark$ & $\checkmark$ & $\checkmark$ & $\checkmark$ & $\checkmark$ & - & $\checkmark$ & - & $\checkmark$ & $\checkmark$ & $\checkmark$ & $x$ \\
\hline 4 & $\checkmark$ & $\checkmark$ & $\checkmark$ & $\checkmark$ & $\checkmark$ & - & 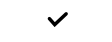 & - & $\checkmark$ & $\checkmark$ & $\checkmark$ & $x$ \\
\hline 5 & $\checkmark$ & $\checkmark$ & $\checkmark$ & $\checkmark$ & $\checkmark$ & $\checkmark$ & $\checkmark$ & - & $\checkmark$ & $\checkmark$ & $\checkmark$ & $\checkmark$ \\
\hline 6 & $\checkmark$ & $\checkmark$ & $\checkmark$ & $\checkmark$ & $\checkmark$ & $\checkmark$ & $\checkmark$ & - & $\checkmark$ & $\checkmark$ & $\checkmark$ & $\checkmark$ \\
\hline 7 & $\checkmark$ & $\checkmark$ & $\checkmark$ & $\checkmark$ & $\checkmark$ & $\checkmark$ & $\checkmark$ & - & $\checkmark$ & $\checkmark$ & $\checkmark$ & $x$ \\
\hline 8 & $r$ & $\checkmark$ & $\checkmark$ & $\checkmark$ & $\checkmark$ & $\checkmark$ & $\checkmark$ & - & $\checkmark$ & $\checkmark$ & $\checkmark$ & $x$ \\
\hline
\end{tabular}

TABLA 2.

Formatos de archivo

\begin{tabular}{|c|c|c|c|c|c|c|c|c|}
\hline Software & RVT & IFC & FBX & OBJ & DAE & GLTF & DWG & Software intermedio \\
\hline 1 & $x$ & $x$ & $\checkmark$ & $x$ & $x$ & $x$ & $x$ & No \\
\hline 3 & $x$ & $x$ & $\checkmark$ & $\checkmark$ & $\checkmark$ & $x$ & $x$ & Sí \\
\hline 4 & $x$ & $\checkmark$ & $\checkmark$ & $x$ & $x$ & $x$ & $x$ & No \\
\hline 6 & $\checkmark$ & $\checkmark$ & $\checkmark$ & $\checkmark$ & $x$ & $\checkmark$ & $\checkmark$ & Sí \\
\hline 7 & $x$ & $x$ & $\checkmark$ & $\checkmark$ & $x$ & $\checkmark$ & $x$ & Sí \\
\hline 8 & $x$ & $x$ & $\checkmark$ & $\checkmark$ & $x$ & $\checkmark$ & $x$ & No \\
\hline
\end{tabular}


TABLA 3.

Opciones básicas del usuario

$$
\text { Software Modificación de capas Implementación de comandos }
$$

\begin{tabular}{|c|c|c|c|c|c|c|c|}
\hline 1 & Pago & $x$ & $x$ & $\checkmark$ & $\checkmark$ & $x$ & $x$ \\
\hline 2 & $x$ & $x$ & $\checkmark$ & $\checkmark$ & $x$ & $x$ & $x$ \\
\hline 3 & $x$ & $x$ & $x$ & $x$ & $\checkmark$ & $x$ & $x$ \\
\hline 4 & $x$ & $x$ & $x$ & $x$ & $\checkmark$ & $x$ & $x$ \\
\hline 5 & $\checkmark$ & $\checkmark$ & $\checkmark$ & $\checkmark$ & $\checkmark$ & $v$ & $\checkmark$ \\
\hline 6 & $\checkmark$ & $\checkmark$ & $\checkmark$ & $\checkmark$ & $\checkmark$ & $\checkmark$ & $\checkmark$ \\
\hline 7 & $\checkmark$ & $\checkmark$ & $\checkmark$ & $\checkmark$ & $\checkmark$ & $\checkmark$ & $x$ \\
\hline 8 & $\checkmark$ & $\checkmark$ & $\checkmark$ & $\checkmark$ & $\checkmark$ & $\checkmark$ & $x$ \\
\hline
\end{tabular}

creación de contenido digital, es el más aceptado por los softwares de realidad aumentada.

La tabla 3 indica las funcionalidades que permite cada software, lo que se traduce en la posibilidad de interacción del usuario. Las funcionalidades que se han analizado han sido: si se permite modificar capas para poder mostrar u ocultar elementos a voluntad; si se permite implementar comandos para interactuar con los elementos; si se permite mover y desplazar el modelo; si se permite rotar y escalar el elemento; si se permite la generación de sombras; si e permite interactuar con los elementos de algún otro modo; y si permite incorporar y utilizar la información BIM. Nuevamente, se ha empleado el mismo sistema de numeración de softwares y de calificación que para las tablas anteriores.

La tabla 4 muestra los tiempos obtenidos tras analizar cada programa al utilizar el modelo de prueba descrito anteriormente, donde: $\mathrm{Tl}$, 1 es el tiempo empleado para el aprendizaje del software; T1,2 es el tiempo empleado para generar el proyecto; T2 es el tiempo que la aplicación necesita para generar el modelo; y T3 es el tiempo total, que también tiene en cuenta la búsqueda previa de información y la instalación de programas y aplicaciones.

TABLA 4.

Tiempos de modelado y ejecución

\begin{tabular}{ccccc}
\hline Software & $\begin{array}{c}\mathrm{T}_{1,1} \\
\text { [hours] }\end{array}$ & $\begin{array}{c}\mathrm{T}_{1,2} \\
\text { [hours] }\end{array}$ & $\begin{array}{c}\mathrm{T}_{2} \\
\text { [seconds] }\end{array}$ & $\begin{array}{c}\mathrm{T}_{3} \\
\text { [hours] }\end{array}$ \\
\hline $\mathbf{1}$ & 0.50 & 0.05 & 7 & 2 \\
$\mathbf{2}$ & 0.75 & 0.25 & 2 & 2.5 \\
$\mathbf{3}$ & 0.75 & 0.09 & 2 & 2.5 \\
$\mathbf{4}$ & 0.75 & 0.09 & 10 & 2.5 \\
$\mathbf{5}$ & 150 & 150 & 2 & 320 \\
6 & 150 & 200 & 2 & 370 \\
7 & 6 & 2 & 7 & 9 \\
8 & 5 & 2 & 3 & 8 \\
\hline
\end{tabular}

En la tabla anterior se aprecia cómo tanto para Unity como para Unreal se necesita de mucho tiempo de aprendizaje y de trabajo para generar el modelo deseado de realidad aumentada. Esto se debe a que ambos son programas diseñados para el de- sarrollo de videojuegos, lo que hace de ellos herramientas muy potentes pero muy complejas de utilizar.

La tabla 5 expresa unas conclusiones finales globales en las que se tiene en cuenta todo lo analizado anteriormente. En esta tabla se evalúan la complejidad, calidad del modelo e interacción en una escala del 0 al 5 , donde 0 implica nulidad y 5 la máxima satisfacción. La columna de complejidad tiene en cuenta todo el proceso de desarrollo de la aplicación de realidad aumentada, desde la búsqueda de información hasta la instalación de la aplicación de RA. La columna de calidad del modelo tiene en cuenta el nivel de detalle del resultado obtenido y el tiempo necesario para ejecutarlo. La columna de interacción evalúa el grado de interacción que tiene el usuario con el modelo. Finalmente, se ha elaborado una columna evaluando las tres anteriores, para dar una nota final a cada software. Esta última columna se ha realizado ponderando de forma global los criterios evaluados.

TABLA 5.

Valoración final

Software Complejidad Calidad del modelo Interacción Evaluación final

\begin{tabular}{llllc}
\hline \multicolumn{1}{c}{ ARki } & 1 & 4 & 1 & 2 \\
AUGmentecture & 2 & 4 & 2 & 2.5 \\
Aumentaty & 1 & 4 & 0 & 2 \\
UniteAR & 1 & 3 & 0 & 1.5 \\
Unity & 5 & 5 & 5 & 5 \\
Unreal Engine & 5 & 5 & 5 & 5 \\
WakingApp & 3 & 5 & 4 & 4 \\
Zappar & 3 & 5 & 4 & 4 \\
\hline
\end{tabular}

En función de lo anterior, se ha obtenido que Unity y Unreal son los programas que mejor se adecuan a lo perseguido en la investigación. Esto se debe, a que son los únicos que permiten la importación de información BIM, pero también a que son los que garantizan una mayor interacción del usuario, lo que se traduce en un mayor número de opciones y empleabilidad. De entre estos, se ha optado por Unity para construir la aplicación de realidad aumentada del puente por haber sido su utilización ligeramente más sencilla, además contar con mayor información y foros de usuarios. 
3.

\section{REALIDAD AUMENTADA APLICADA AL PROYECTO DE UN PUENTE REAL}

\subsection{Descripción de la estructura}

El puente empleado en esta investigación es un puente real localizado en Belleville, en el condado de St. Clair, Illinois, EEUU. Los planos e información sobre este puente han sido facilitados por Saint Louis University.

Este puente está compuesto en realidad por cuatro estructuras, pero solo una de ellas ha sido empleada para la aplicación de RA por el gran tamaño de archivo que suponía modelar las cuatro. La estructura empleada es la nombrada como SN1 17 por el Departamento de Transportes del Estado de Illinois, y puede apreciarse en la Figura 2. Esta estructura se conforma de estribos, una pila, 5 filas de vigas de acero unidas con cruces de San Andrés y un tablero compuesto de una losa y barreras. El puente tiene una longitud de $107 \mathrm{~m}$ y cuenta con una pendiente ascendente constante (zona izquierda de la imagen) del $3.23 \%$ a lo largo de $62 \mathrm{~m}$ y una pendiente descendiente constante (zona derecha de la imagen) del $-4.07 \%$ a lo largo de $45 \mathrm{~m}$.

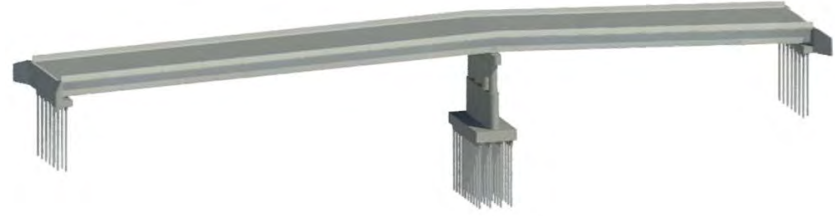

Figura 2. Vista 3D del puente SN117

\subsection{Aplicación de realidad aumentada}

Tras realizar el modelo del puente con Revit, se ha importado en Unity utilizando el plug-in PXYZ para garantizar la importación de la información BIM. Posteriormente se ha trabajado con dicho programa para generar el modelo de RA. Finalmente se ha desarrollado la aplicación móvil que contiene el modelo y se ha ejecutado en un dispositivo móvil. Los pasos generales seguidos en este proceso son:

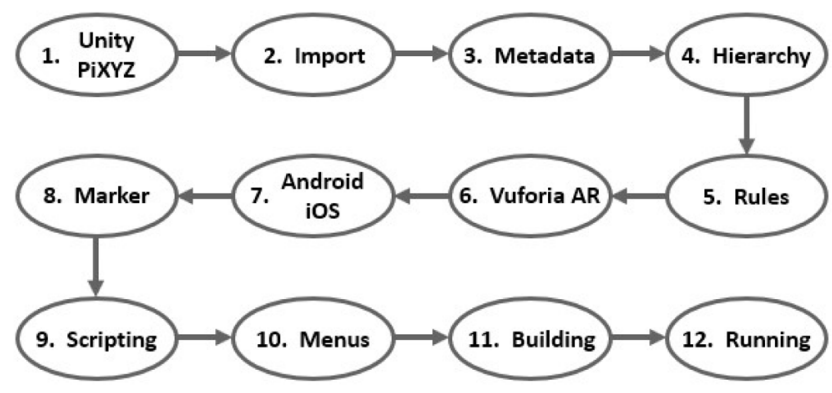

Figura 3. Proceso de elaboración de la app de RA

El proceso de elaboración de la aplicación de realidad aumentada se ha dividido en 12 pasos: el primer paso (1. Unity/PiXYZ) consiste en la búsqueda de información, descarga de Unity y el plug-in PiXYZ, instalación de ambos y ajuste de la configuración necesaria para adecuar el escenario de trabajo al deseado por el proyecto; en segundo lugar (2. Import) se procede a la importación del modelo 3D del puente, elaborado con Revit y exportado a IFC; en el tercer paso (3. Metadata) se comprueba que la información BIM ha sido correctamente importada para todos los elementos; en cuarto lugar (4. Hierarchy) se reordena la jerarquía del modelo para poder trabajar más fácilmente y para poder ejecutar posteriormente comandos que afectarán a esta jerarquía, por ejemplo, para mostrar u ocultar elementos; el quinto paso (5. Rules) consiste en la creación de reglas para mejorar la visualización, como centrar el modelo en el origen de coordenadas o definir todos los elementos como rígidos para evitar ser atravesados por la cámara cuando el usuario interactúe con ellos; en sexto lugar (6. Vuforia AR) se activa la herramienta de Vuforia, que será la encargada de realizar el modelo de realidad aumentada, y se establecen los ajustes necesarios en la configuración para poder construir y ejecutar la aplicación sin errores; el séptimo paso (7. Android/ iOS) es elegir en qué sistema operativo se trabajará con la app de RA, y configurar los ajustes para trabajar en este entorno; en octavo lugar (8. Marker) se crea un marcador, que será leído por la app para situar el modelo del puente encima, se solicita su licencia de uso a Vuforia, se incorpora al programa y se realizan las operaciones necesarias para su correcto funcionamiento; en el noveno paso (9. Scripting) se escriben los códigos para los comandos que se van a desear para la interacción del usuario con el modelo, por ejemplo, para mover, rotar, escalar el puente $\mathrm{u}$ obtener la información BIM de los elementos; en décimo lugar (10. Menus) se crean distintos menús, uno para gestionar la visibilidad de las capas y otro para seleccionar qué acción de interacción se quiere realizar, que facilitarán al usuario el manejo de la aplicación; el undécimo paso (11. Building) consiste en la creación de la app de RA y la instalación en el dispositivo donde se usará; el duodécimo, y último, paso (12. Running) es la ejecución de la aplicación y su utilización.

Para este proyecto, la aplicación ha sido diseñada para versiones de Android 7.0 o posterior, y ha sido probada en un dispositivo Xiaomi Mi Al. A continuación, se muestran una serie de capturas de pantalla de la aplicación:

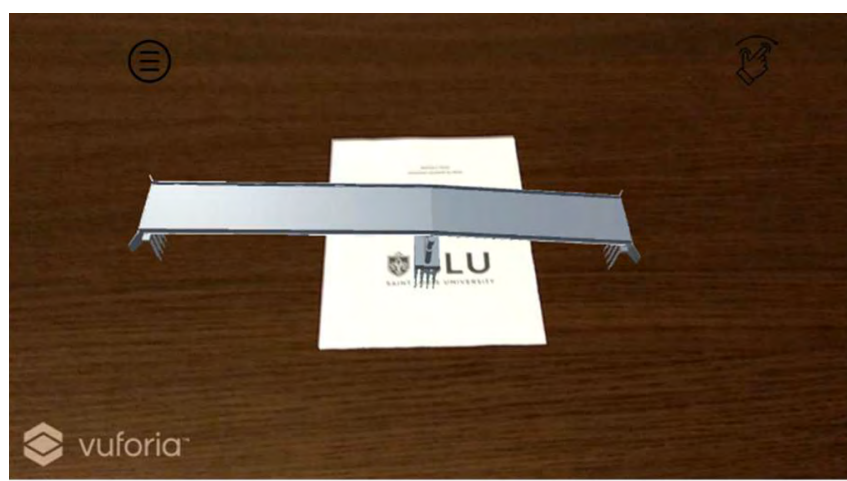

Figura 4. Vista inicial de la aplicación

La imagen anterior representa lo mostrado por la aplicación tras su ejecución y tras leer el marcador. Es el punto de partida tal y como se ha establecido en Unity. 


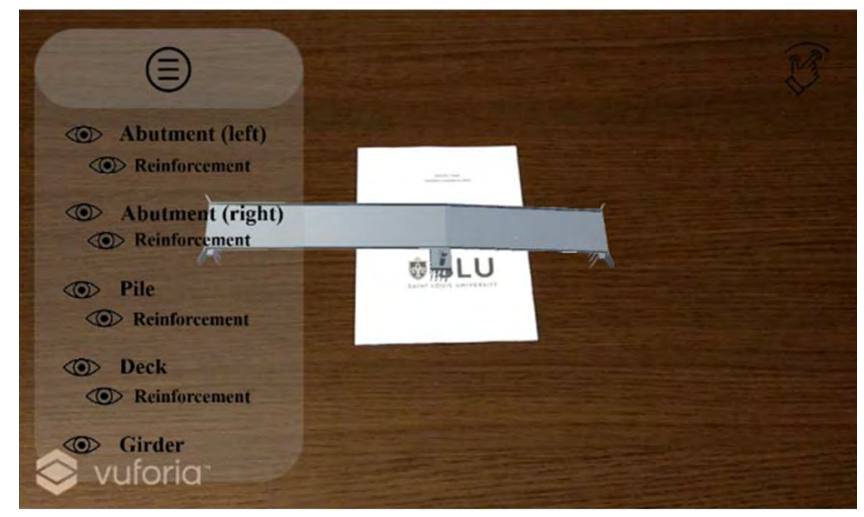

Figura 5. Menú de visibilidad

La ilustración anterior muestra el menú de visualización desplegable, donde se pueden activar o desactivar capas para visualizar $\mathfrak{u}$ ocultar, respectivamente, distintos elementos.

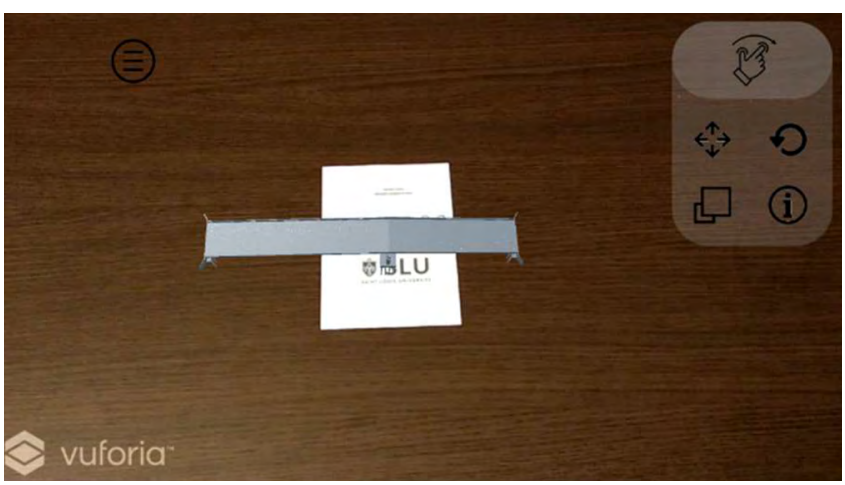

Figura 6. Menú de gestos

La imagen anterior muestra el menú de gestos disponibles para el usuario: mover, rotar, escalar el puente u obtener la información BIM asociada al elemento seleccionado. Estos comandos de interacción han sido incluidos en un menú para facilitar el manejo de la aplicación y evitar realizar acciones indeseadas.

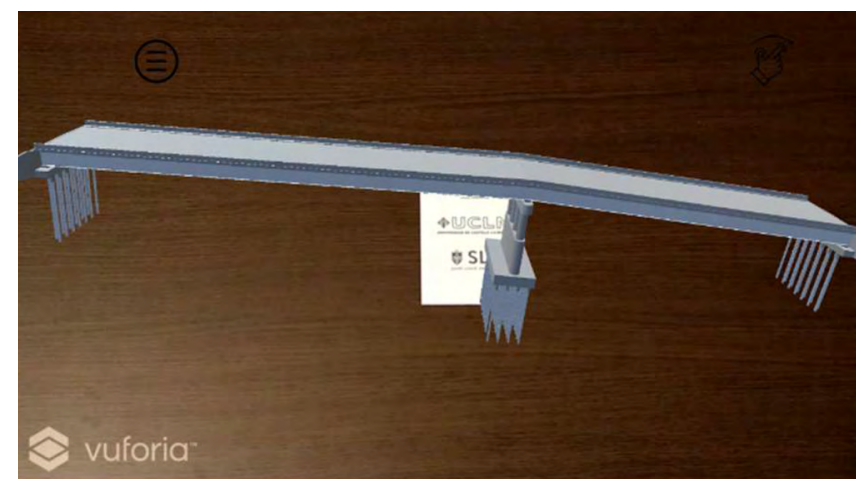

Figura 7. Puente rotado y ampliado

En la ilustración anterior se muestra una vista del puente tras interactuar con él, específicamente tras ser rotado y escalado.

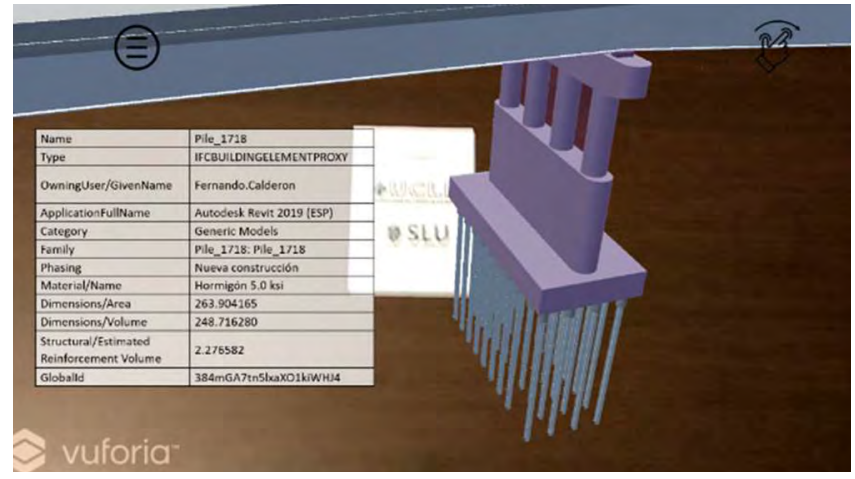

Figura 8. Información BIM de la pila

La imagen anterior muestra la información BIM asociada a la pila del puente, la cual ha sido resaltada en violeta tras ser seleccionada, para facilitar al usuario reconocer qué elemento ha seleccionado y así evitar posibles errores.

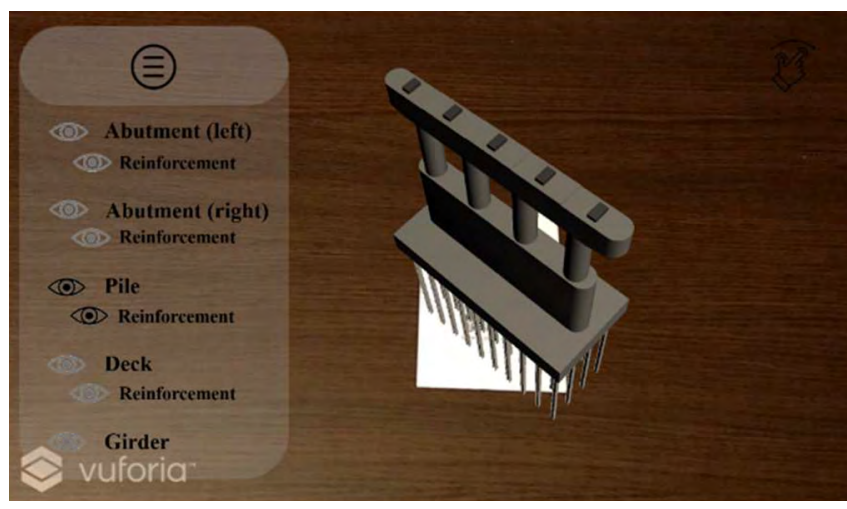

Figura 9. Pila aislada

La ilustración anterior muestra la pila del puente únicamente tras haber desactivado la visualización del resto de elementos.

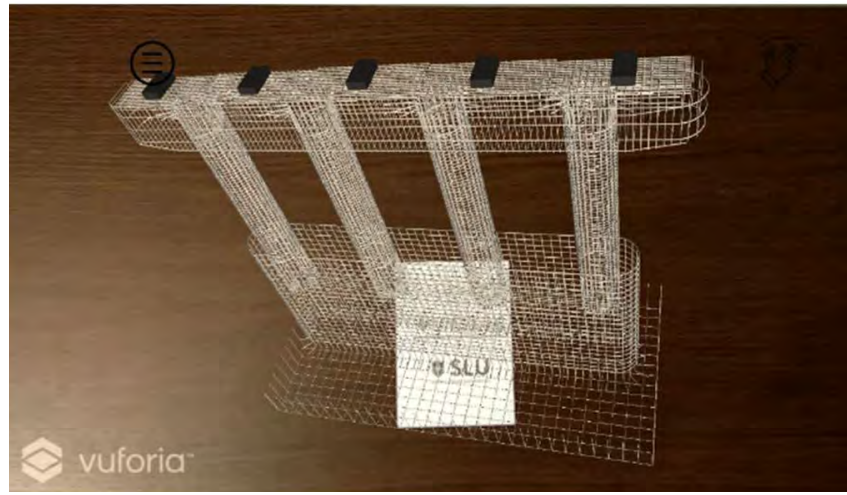

Figura 10. Armadura de la pila aislada

La imagen anterior muestra de manera aislada la armadura de la pila. 


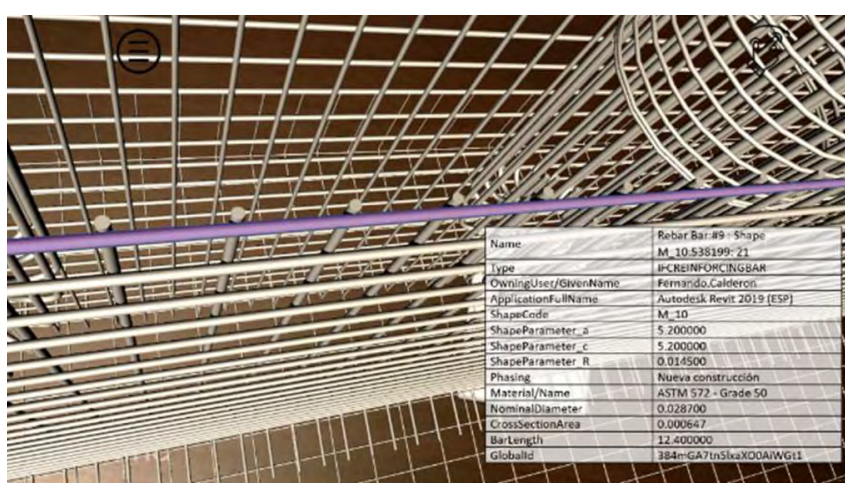

Figura 11. Información BIM de una barra

La ilustración anterior muestra la información BIM asociada a una de las barras que componen la armadura de la pila, en violeta.

\section{4.}

\section{FUTURAS INVESTIGACIONES}

Para el análisis de los distintos softwares de realidad aumentada se empleó un modelo de pequeña escala elaborado con un único software: Revit. Sin embargo, en ingeniería civil se desarrollan proyectos de escalas muy distintas, y casi siempre se emplea más de un software en su elaboración, por lo que algunas de las futuras investigaciones que se podrían hacer son: 1) repetir el análisis de programas de RA con modelos de mediana y gran escala para estudiar las diferencias; 2) analizar qué softwares BIM permiten exportaciones a FBX, ya que es el archivo más utilizado por aplicaciones de RA, y estudiar el flujo de trabajo de los softwares que no permiten su exportación; 3) analizar el flujo de trabajo cuando se modela con más de un software BIM.

Dado que los programas que permiten importar información BIM y gran interacción del usuario son pensados para el desarrollo de videojuegos, utilizarlos para la ingeniería civil hace que sean complicados de manejar, con lo que otra posible línea de investigación futura podría ser la creación de un programa que permitiese importar archivos en IFC, ya que todos los softwares BIM permiten trabajar con este formato, y que contase con opciones de interacción preprogramadas para mover, rotar, escalar, ocultar/mostrar elementos y obtener información del modelo. Esto facilitaría mucho la implementación de RA en la ingeniería civil.

\section{5.}

\section{CONCLUSIONES}

La primera conclusión que se extrae tras esta investigación es que tanto la metodología BIM como la tecnología de RA son dos herramientas que presentan un gran potencial pero que se encuentran aún en desarrollo, y se debe invertir más tiempo y recursos en estudiar su interacción e implementación en la ingeniería civil.
Otra de las conclusiones principales es que generar modelos de RA con información BIM es bastante complejo, y hay que recurrir a programas de desarrollo de videojuegos para ello. Implementar esto en una empresa requeriría una gran inversión económica y de tiempo, ya que no son softwares intuitivos y requiere de una formación especializada para trabajar con ellos, pero a la larga serían beneficiosos para ella.

Tanto Unity como Unreal, ambos softwares para desarrollo de videojuegos, son herramientas muy potentes que permiten infinidad de posibilidades de interacción y formas de aplicarlas. Como se ha comprobado, la tecnología actual permite vincular modelos elaborados con BIM con herramientas de realidad aumentada. Sin embargo, aún queda mucho que investigar sobre su implementación en la ingeniería civil, por lo que el proyecto desarrollado, y por tanto el presente artículo, tienen entre sus objetivos el inspirar a otros investigadores a estudiar sobre esta interacción de BIM y realidad aumentada.

Tras utilizar la aplicación de realidad aumentada elaborada para el puente, se ha llegado a la conclusión de que su mayor potencial podría darse durante la fase de explotación y mantenimiento de los puentes. A pesar de esto, emplear RA podría ser también beneficioso para las fases de diseño y construcción del puente, ya que mejora la visualización y comprensión de la estructura, y facilita la obtención de información de los elementos.

Además de todo lo anterior, el empleo de la realidad aumentada en ingeniería civil es una manera más atractiva y dinámica de vender y defender un proyecto, lo cual se viene haciendo desde hace tiempo en otros sectores como la arquitectura.

\section{Agradecimientos}

Los autores agradecen a todas las personas que han ayudado a desarrollar esta investigación, en particular a la Universidad de Castilla-La Mancha y a Saint Louis University.

\section{Referencias}

[1] Calderón de Diego, F. (2019). Augmented reality technology in BIM projects: application to bridges (Trabajo de Fin de Máster por la Universidad de Castilla-La Mancha). Recuperado de app-uclmsky-webs-ingcaminos.azurewebsites.net

[2] Diao, P.H. \& Shis, N.J. (2019). "BIM-Based AR Maintenance System (BARMS) as an Intelligent Instruction Platform for Complex Plumbing Facilities". International Journal of Applied Sciences, 9 (8), 1592. DOI: 10.3390/app9081592

[3] El Ammari, K. \& Hammad, A. (2019). "Remote interactive collaboration in facilities management using BIM-based mixed reality". Automation in Construction, 107 (in progress). DOI: 10.1016/j.autcon.2019.102940

[4] Love, E.D., Zhou, J. \& Matthews, J. (2019). "Project controls for electrical, instrumentation and control systems: Enabling role of digital system information modelling". Automation in Construction, 103, pp 202-212. DOI: 10.1016/j.autcon.2019.03.010

[5] Shin, D. \& Dunston, P. (2008). "Identification of application areas for Augmented Reality in industrial construction based on technology suitability". Automation in Construction, 17 (7), pp 882-894. DOI: 10.1016/j.autcon.2008.02.012 
[6] Nassereddine, H., Veeramani, D. \& Hanna, A. (2019). "Augmented reality-enabled production strategy process". 36th International Symposium on Automation and Robotics in Construction, Banff, Alberta, Canada, pp 297-305. DOI: 10.22260/ISARC2019/0040

[7] Wang, X. \& Dunston, P.S. (2006). "Potential of augmented reality as an assistant viewer for computer-aided drawing". Journal of Computing in Civil Engineering, 20 (6), pp 437-441. DOI: 10.1061/(ASCE)08873801(2006)20:6(437)

[8] Schweigkofler, S., Monizza, G.P., Domi, E., Popescu, A., Ratajzak, J., Marcher, C., Riedl, M. \& Matt, D. (2018). "Development of a Digital Platform Based on the Integration of Augmented Reality and BIM for the Management of Information in Construction Processes". IFIP In ternational Federation for Information Processing, 540, pp 46-55. DOI 10.1007/978-3-030-01614-2 5

[9] Baek, F., Ha, I. \& Kim, H. (2019). "Augmented reality system for facility management using image-based indoor localization". Automation in Construction, 99, pp 18-16. DOI: 10.1016/j.autcon.2018.11.034

[10] Nandavar, A., Petzold, F., Schubert, G. \& Youssef, E. (2019). "Opening BIM in a new dimension". 24 th International Conference on Computer-Aided Architectural Design Research in Asia, CAADRIA, 1, pp 595604. Retrieved from scopus.com 